\title{
O Efeito Casimir em Teoria das Fontes
}

\section{The Casimir effect in Source Theory}

\author{
F. A. Barone ${ }^{1}$, A. A. Nogueira*2, B. M. Pimentel ${ }^{2}$ \\ ${ }^{1}$ Instituto de Física e Química, Universidade Federal de Itajubá, Itajubá, MG, Brasil \\ ${ }^{2}$ Instituto de Física Teórica, Universidade Estadual Paulista, São Paulo, SP, Brasil
}

Recebido em 7 de fevereiro de 2016. Revisado em 19 de maio de 2016. Aceito em 22 de maio de 2016

Este trabalho tem como objetivo introduzir a interpretação variacional de Schwinger ao fenômeno de Casimir, associada à construção de uma dinâmica quântica que descreva efetivamente a interação entre objetos macroscópicos (2 fontes) do ponto de vista de uma teoria de espalhamento (Matriz-S), utilizando como guia fenomenológico a interação entre meios materiais. Com este intuito estudaremos a imposição de condições de contorno no campo escalar real de Klein-Gordon-Fock não massivo em $(1+1)$ dimensões no formalismo de operadores e no formalismo funcional. Tendo elucidado o método funcional, abordaremos o problema utilizando a leitura de Schwinger.

Palavras-chave: Teoria Clássica de Campos; Teoria Quântica de Campos.

The aim of this work is to introduce the Schwinger interpretation to the Casimir phenomenon, associated with the construction of a quantum dynamics which effectively describes the interaction between macroscopic objects (sources) from the point of view of a scattering theory (S-Matrix) using as guide the phenomenological interaction in material means. With this intention let's study the imposition of bondary conditions to the real non-massive Klein-Gordon-Fock field in $(1+1)$ dimensions in the operatorial formalism and in the funcional formalism. Once elucidated the functional method, we approach the problem using the Schwinger reading.

Keywords: Classical Field Theory; Quantum Field Theory.

\section{Introdução ao fenômeno}

Em meados de 1948, após ter investigado os efeitos associados a força de London-van der Waals [1] na explicação da estabilidade de grande agregados de átomos ou suspensões, o físico Holandês Hendrik Casimir do Laboratório de Pesquisa Phillips previu que duas placas metálicas paralelas descarregadas estão sujeitas a uma força tendente a aproximá-las [2,3]. O interessante é que essa previsão foi consequência de discussões com o físico Niels Bohr que havia feito uma observação de que os trabalhos de Casimir associados à modificação dos modos do campo eletromagnético devido às condições de contorno, pareciam estar ligados à energia de ponto zero ou melhor a energia de vácuo. Essa era a pista que Casimir tanto procurava para encontrar um argumento

\footnotetext{
*Endereço de correspondência: andsogueira@hotmail.com
}

físico simples que complementasse a estrutura matemática já obtida. Essa força somente é mensurável quando a distância entre as duas placas é extremamente pequena, da ordem de $1 \mu \mathrm{m}$. O efeito poderia ser interpretado pelo fato do espaço vazio ter flutuações do vácuo devido ao princípio da incerteza de Heisenberg.

A fenomenologia associada à álgebra dos operadores de criação e destruição nos levaria a concluir que a força de Casimir estaria associada à criação e destruição de partículas virtuais não satisfazendo o princípio da conservação de energia e momento. Históricamente, esse fato levou a uma discussão em termos de uma pressão virtual, pois o espaço entre as duas placas restringe o alcance dos comprimentos de onda possíveis para as partículas virtuais de 
tal forma que poucas delas estão presentes entre as placas. Como resultado, há uma menor densidade de energia entre as duas placas do que no espaço fora dessa região. Em essência, há mais partículas virtuais fora do que dentro da região entre as placas, criando uma diferença de pressão e, consequentemente, uma força. Um efeito análogo foi observado por marinheiros franceses no século XVIII. No caso, dois navios balançam de um lado a outro com fortes marés e ventos fracos. Os navios se aproximam devido à interferência destrutiva entre os navios eliminar a maré nessa região. O mar calmo entre os navios tem uma densidade de energia menor que a maré de cada lado dos navios criando uma pressão que pode empurrar os navios para mais perto 44. Porém, sabemos hoje em dia que a dicussão em termos de uma pressão é equivocada devido ao fato de existirem condições de contornos como, por exemplo, o de placas mistas (uma placa condutora e outra infinitamente permeável), que nos dão uma força repulsiva com modos do campo discretizados entre as placas. Novamente, ao se estudar o efeito Casimir em uma linguagem funcional e aplicarmos a fenomenologia de partículas de Stueckelberg-Feynmann perceberíamos que a força de Casimir estaria associada às funções de Green avaliadas no mesmo ponto, diagrama bolha. Na representação de coordenadas teríamos a criação e destruição de uma partícula no mesmo ponto do espaço e na representação de momentos essa criação e destruição de partícula teria momento e energia arbitrários, sendo, portanto, virtual. Pois bem, em Teoria Quântica de Campos a interação é dada por uma hamiltoniana de interação com ordenamento normal, : $H_{\text {int }}:$, onde o vácuo não possui uma dinâmica virtual. Sendo assim uma pergunta natural seria como implementar uma leitura da interação entre as placas, anteriormente dadas em termos das condições de contorno, em termos de uma dinâmica quântica de campos real.

O efeito Casimir foi observado experimentalmente em 1997 por Lamoreaux [5] do Laboratório Nacional de Los Alamos e colaboradores, apesar de Sparnaay [6] ter demonstrado experimentalmente apenas a existência do efeito atrativo. Na prática, em vez de usar duas placas paralelas que requeriria um alinhamento perfeitamente acurado, o experimento de Lamoreaux usa uma placa plana e outra placa que é parte de uma esfera com um grande raio de curvatura. Vemos também que no procedimento experimental a balança de torção é reformulada com dispositivos eletrônicos a fim de abarcar a precisão necessária nas medidas experimentais. Porém, durante os últimos 15 anos o efeito Casimir foi medido com grande precisão por Mohideen e Decca [7].

O experimento de Mohideen e Decca demonstra o contra-intuitivo fenômeno quântico de que o vácuo de fato tem uma energia associada a ele, a energia de ponto zero, apesar de o vácuo não possuir uma dinâmica real, mas sim virtual. O estudo de campos eletromagnéticos em meios materiais atribuído a Lifshitz [8] abriu as portas para uma nova interpretação ao fenômeno. Nessa interpretação do fenômeno, a força de Casimir está associada a uma interação efetiva entre correntes no contexto de Eletrodinâmica Quântica, pois as placas têm como estrutura microscópica distribuições probabilísticas de cargas que em média são nulas, são meios materiais. A formulação microscópica do problema de interação basea-se no estudo e solução das equações de Heisenberg em meios materiais. Macroscopicamente, essas correntes randômicas dão origem a forças retardadas de van der Waals 9 12. Schwinger inspirou-se, fenomenologicamente, na interação entre meios materiais para construir uma interpretação funcional para o efeito Casimir (Teoria das Fontes) [13], construindo uma dinâmica de correntes (2 fontes) que representa efetivamente a interação entre as placas condutoras do ponto de vista de um método variacional em teoria de espalhamento (Matriz-S). A busca por uma dinâmica quântica de campos que descreva efetivamente a força de Casimir também pode ser vista em [14 16], em que vemos a condição de contorno (Dirichlet) sendo moldada em termos de uma lagrangiana de interação com campos externos. Em suma, procuramos uma dinâmica para a teoria microscópica fenomenológicamente estudando a física da interação em meios materiais e, posteriormente, descrevemos a teoria macroscópica efetivamente.

Considerando a extensa literatura e suas aplicações 17 23, explícitaremos e comentaremos alguns tópicos. Podemos implementar outros tipos de condições de contorno menos idealizadas no problema para possivelmente descrever uma situação real como superfícies permeáveis, dielétricas e com rugosidade. Também podemos estudar o problema em várias configurações de borda como superfícies cilíndricas e esféricas, encontrando assim a influência de efeitos de tamanho, topologia e geometria da borda sobre a força de Casimir. Outro exemplo é a influência do movimento da condição de contorno 
sobre a energia de Casimir e a criação de fótons do vácuo análogo ao efeito que acontece quando aplicamos um campo externo 25. É possível encontrar correções no espectro de um átomo quando este está entre duas placas condutoras perfeitas. Encontramos também efeitos associados à variação da velocidade da luz quando esta se propaga entre as duas placas condutoras perfeitas conhecida como efeito Scharnhorst [26]. Na eletrodinâmica generalizada de Podolsky [27] também vemos o cálculo de correções ao efeito Casimir e, assim, se sucede a vasta possibilidade de se explorar o fenômeno.

\section{O Efeito Casimir no formalismo de operadores}

Com o objetivo de simplificar o problema, consideremos um campo relativístico livre de massa nula em $(1+1)$ dimensões. O movimento deste campo é governado pela equação de Einstein ${ }^{1}$

$$
E^{2}=p^{2} .
$$

Tendo em vista o princípio da correspondência

$$
\begin{aligned}
& E \rightarrow i \frac{\partial}{\partial t} \\
& p \rightarrow-i \frac{\partial}{\partial x} .
\end{aligned}
$$

a equação que descreve esse campo é a seguinte

$$
\left[\frac{\partial^{2}}{\partial t^{2}}-\frac{\partial^{2}}{\partial x^{2}}\right] \phi(t, x)=0
$$

onde o campo em questão é conhecido como campo escalar de Klein-Gordon-Fock (KGF).

Supondo que exista um objeto físico que anule o campo em determinados pontos do espaço-tempo passaremos a impor as condições de contorno, ou melhor, escolheremos a topologia

$$
\phi(t, 0)=0=\phi(t, a) .
$$

Essas condições de contorno são uma idealização do plano condutor perfeito.

Observando que a equação que descreve as propriedades do campo é uma equação de onda, podemos utilizar o método de separação de variáveis para resolver a equação diferencial parcial (3)

$$
\phi(t, x)=T(t) X(x)
$$

e conseqüentemente temos a seguinte solução

$$
\phi(t, x)=e^{i \omega t} \operatorname{sen}(k x)
$$

onde $\omega= \pm k$.

Utilizando as condições de contorno encontramos que

$$
k_{n}=\frac{n \pi}{a}, \quad n=1,2,3, \ldots
$$

Portanto, concluímos que o espectro de energia (frequência) do campo entre as placas é discreto

$$
E_{n}=k_{n}=\omega_{n}=\frac{n \pi}{a} .
$$

Como a equação de onda é linear, a solução geral do nosso problema é dada por uma combinação linear

$$
\begin{aligned}
& \phi(x, t)=\sum_{n=1}^{\infty}\left[e^{-i \omega_{n} t} \operatorname{sen}\left(k_{n} x\right) a^{-}{ }_{n}\right. \\
& \left.+e^{i \omega_{n} t} \operatorname{sen}\left(k_{n} x\right) a^{+}{ }_{n}\right] .
\end{aligned}
$$

onde $a^{-}$é o coeficiente associado à frequência positiva e $a^{+}$é o coeficiete associado à frequência negativa. Por simplicidade, impomos que o campo escalar seja real

$$
\left(a^{-} n\right)^{*}=a^{+}{ }_{n} .
$$

Agora, para quantizarmos o campo, elevamos os coeficientes da expansão anterior em nível de operadores

$$
\begin{aligned}
& a^{-}{ }_{n} \rightarrow \hat{a}_{n} \\
& a^{+}{ }_{n} \rightarrow \hat{a}_{n}^{\dagger}
\end{aligned}
$$

$$
\left.\hat{\phi}(x, t)=\sum_{n=1}^{\infty} e^{-i \omega_{n} t} \operatorname{sen}\left(k_{n} x\right) \hat{a}_{n}+e^{i \omega_{n} t} \operatorname{sen}\left(k_{n} x\right) \hat{a}_{n}^{\dagger}\right]
$$

e interpretamos $\hat{a}_{n}$ como operador de destruição e $\hat{a}_{n}^{\dagger}$ como operador de criação, satisfazendo as propriedades

$$
\begin{aligned}
& {\left[\hat{a}_{n}, \hat{a}_{n^{\prime}}^{\dagger}\right]=i \delta_{n, n^{\prime}}, \quad\left[\hat{a}_{n}, \hat{a}_{n^{\prime}}\right]=0, \quad\left[\hat{a}_{n}^{\dagger}, \hat{a}_{n^{\prime}}^{\dagger}\right]=0} \\
& \hat{a}_{n}|0\rangle=0 .
\end{aligned}
$$

Para encontrar a energia desse sistema físico basta escrevermos a Hamiltoniana

$$
\mathcal{H}=\pi \dot{\phi}-\mathcal{L}, \quad \pi=\frac{\partial \mathcal{L}}{\partial \dot{\phi}},
$$

\footnotetext{
${ }^{1}$ Estamos trabalhando no sistema natural de unidades em que $c=1=\hbar$.
} 


$$
\begin{aligned}
\mathcal{L} & =\frac{1}{2}\left[\partial_{\mu} \phi \partial^{\mu} \phi\right]=\frac{1}{2}\left[\left(\partial_{t} \phi\right)^{2}-\left(\partial_{x} \phi\right)^{2}\right], \\
\mathcal{H} & =\frac{1}{2}\left[\left(\partial_{t} \phi\right)^{2}+\left(\partial_{x} \phi\right)^{2}\right],
\end{aligned}
$$

e quantizar

$$
\begin{aligned}
& \mathcal{H} \rightarrow \hat{\mathcal{H}}=\frac{1}{2}\left[\left(\partial_{t} \hat{\phi}\right)^{2}+\left(\partial_{x} \hat{\phi}\right)^{2}\right] \\
& =\frac{1}{2}\left[\sum_{n=1}^{\infty}-i \omega_{n}\left(e^{-i \omega_{n} t} \operatorname{sen}\left(k_{n} x\right) \hat{a}_{n}\right.\right. \\
& \left.\left.-e^{i \omega_{n} t} \operatorname{sen}\left(k_{n} x\right) \hat{a}_{n}^{\dagger}\right)\right]^{2} \\
& +\frac{1}{2}\left[\sum _ { n = 1 } ^ { \infty } k _ { n } \left(e^{-i \omega_{n} t} \cos \left(k_{n} x\right) \hat{a}_{n}\right.\right. \\
& \left.\left.+e^{i \omega_{n} t} \cos \left(k_{n} x\right) \hat{a}_{n}^{\dagger}\right)\right]^{2} .
\end{aligned}
$$

Dando continuidade, podemos definir a energia de ponto zero, $E^{1} T$, ou energia de vácuo entre as duas placas como sendd ${ }^{2}$

$$
\begin{aligned}
E_{T}^{1} & =\int_{0}^{a}\langle 0|\hat{\mathcal{H}}(x)| 0\rangle d x \\
& =\frac{1}{2} \sum_{n=1}^{\infty} \omega_{n}=\frac{1}{2} \frac{\pi}{a} \sum_{n=1}^{\infty} n .
\end{aligned}
$$

que é, nada mais nada menos, que uma soma sobre todas as possibilidades do espectro da partícula entre as placas a menos de um fator $\frac{1}{2}$. Esse fator advém do fato do campo escalar representar um conjunto infinito de osciladores harmônicos, em cada ponto do espaço temos vibrações de um oscilador harmônico. Ao interpretarmos o problema, observando o espectro discreto, vemos uma analogia com Planck e seu estudo sobre cavidades e radiação de corpo negro. Os processos que contribuem para a energia de vácuo são aqueles os quais a partícula é criada e destruída instantaneamente com uma dada energia e momento, o que não satisfaz o princípio da conservação de energia-momento, logo, são ditos processos virtuais.

Como podemos ver a equação anterior é divergente. Com a finalidade de separar a divergência da equação anterior utilizaremos um procedimento chamado regularização

$$
\begin{aligned}
E^{1} T & =\frac{\pi}{2 a} \lim _{\delta \rightarrow 0} \sum_{n=1}^{\infty} n e^{-\delta n} \\
& =-\frac{\pi}{2 a} \lim _{\delta \rightarrow 0} \frac{d}{d \delta} \sum_{n=1}^{\infty} e^{-\delta n}
\end{aligned}
$$

Este procedimento revela que a altas energias o plano condutor perfeito passa a ser transparent $3^{3}$ ou seja, o procedimento tem um significado físico e não depende da função reguladora. Estamos lidando com uma série geométrica de razão $q=e^{-\delta}$ e desse modo

$$
\begin{aligned}
& E^{1}{ }_{T}=-\frac{\pi}{2 a} \lim _{\delta \rightarrow 0} \frac{d}{d \delta}\left[\frac{e^{-\delta}}{\left(1-e^{-\delta}\right)}\right]= \\
& -\frac{\pi}{2 a} \lim _{\delta \rightarrow 0} \frac{d}{d \delta}\left[\frac{1}{\left(e^{\delta}-1\right)}\right] .
\end{aligned}
$$

Como $\delta$ é uma quantidade pequena,

$$
\begin{aligned}
& \frac{d}{d \delta}\left[\frac{1}{\left(e^{\delta}-1\right)}\right]=-\frac{e^{\delta}}{\left(e^{\delta}-1\right)^{2}}=\frac{-1}{4\left[\operatorname{senh}\left(\frac{\delta}{2}\right)\right]^{2}} \cong \\
& \cong \frac{-1}{4\left[\frac{\delta}{2}+\frac{\delta^{2}}{2.12}\right]^{2}}=-\frac{1}{\delta^{2}} \frac{1}{\left[1+\frac{\delta^{2}}{12}\right]^{2}} \\
& \cong\left[-\frac{1}{\delta^{2}}+\frac{1}{12}+O\left(\delta^{2}\right)\right] .
\end{aligned}
$$

Portanto,

$$
E_{T}^{1} \cong-\frac{\pi}{2 a}\left[-\frac{1}{\delta^{2}}+\frac{1}{12}+O\left(\delta^{2}\right)\right] .
$$

Por outro lado, quando afastamos infinitamente as placas, podemos tomar o limite do espectro de energia para o contínuo e definir a energia entre as placas nessa configuração, $E^{2} T$, como sendd 4

$$
E^{2} T=\int \omega(n) d n=\frac{\pi}{2 a} \int_{0}^{\infty} n d n .
$$

Sendo assim,

$$
E^{2} T=-\frac{\pi}{2 a} \lim _{\delta \rightarrow 0} \frac{d}{d \delta} \int_{0}^{\infty} e^{-\delta n} d n=\frac{\pi}{2 a \delta^{2}} .
$$

Como um sistema físico sempre vai para sua configuração de menor energia, a diferença de energia

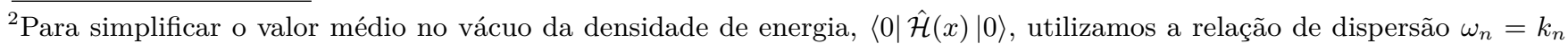
juntamente com o fato de apenas os termos $\hat{a}_{n} \hat{a}_{n}^{\dagger}$ contribuirem para o mesmo, devido as propriedades em 13 .

${ }^{3}$ Observa-se que para um dado $\delta$ infinitesimal, frequências muito altas, $\omega_{n} \gg \omega_{1}$, contribuem muito pouco em (17) devido à exponencial negativa.

${ }^{4}$ A justificativa rigorosa do limite adequado para o contínuo baseia-se na quantização do campo escalar livre de KGF em uma caixa suficientemente grande 24 .
} 
entre as duas configurações das placas é interpretada como o trabalho necessário para separá-las,

$$
\Delta E=\lim _{\delta \rightarrow 0}\left(E_{T}^{2}-E_{T}^{1}\right)=\frac{\pi}{24 a} .
$$

Neste caso a força entre as duas placas é dada por

$$
F_{C}=\frac{d}{d a} \Delta E=-\frac{\pi}{24 a^{2}} .
$$

Trabalhamos no sistema natural de unidades onde $\hbar=1$ e $c=1$. Para escrevermos a força no sistema internacional de unidades, devemos utilizar um pouco de álgebra

$$
[\hbar]^{x} \cdot[c]^{y} \cdot\left[F_{C}\right]^{z}=[F]
$$

onde

$$
\begin{aligned}
& {[\hbar]=[F][L][T]} \\
& {[c]=\frac{[L]}{[T]}} \\
& {\left[F_{C}\right]=\frac{1}{[L]^{2}} .}
\end{aligned}
$$

De imediato concluímos que a força de Casimir é dada por

$$
F_{C}=\frac{d}{d a} \Delta E=-\frac{\pi \hbar c}{24 a^{2}} .
$$

Observa-se que a dependência em $\hbar$ é uma evidência de que o fenômeno ao qual estamos explorando é inteiramente quântico e não existe análogo clássico. $\mathrm{E}$, também, a dependência em $c$ evidencia o caráter relativístico.

\section{O efeito Casimir no formalismo funcional}

Agora construiremos a linguagem funcional necessária para abordar o efeito Casimir [28]. Esta construção será importante para implementar posteriormente a Leitura de Schwinger, pois ganharemos familiaridade com as fontes e as funções de Green (propagadores).

\subsection{O Propagador livre}

O modelo de Klein-Gordon-Fock não massivo em $(1+1)$ dimensões é descrito pela seguinte lagrangeana

$$
\mathcal{L}=\frac{1}{2} \partial_{\mu} \phi \partial^{\mu} \phi+J \phi .
$$

No intuito de implementar uma linguagem quântica, escrevemos o funcional gerador da teoria

$$
Z=\int D \phi \exp [i S]
$$

DOI: http://dx.doi.org/10.1590/1806-9126-RBEF-2016-0033

$$
=\int D \phi \exp \left[i \int d^{2} x \mathcal{L}\right] .
$$

Utilizando o teorema da divergência de Gauss juntamente com o fato do campo $\phi$ se anular nas bordas do espaço-tempo, concluímos que

$$
\begin{gathered}
\int d^{2} x \partial_{\mu} \phi \partial^{\mu} \phi=\int d^{2} x\left[-\phi \square \phi+\partial_{\mu}\left(\phi \partial^{\mu} \phi\right)\right], \\
Z=\int D \phi \exp \left\{-i \int d^{2} x\left[\frac{1}{2} \phi \square \phi-J \phi\right]\right\}, \\
\square=\partial_{\mu} \partial^{\mu} .
\end{gathered}
$$

Como estamos integrando sobre todas as configurações de campo possíveis, podemos fazer uma translação no campo $\phi$ (variável de integração). Por conveniência fazemos

$$
\phi \rightarrow \phi+\int d^{2} y D(x, y) J(y) .
$$

em que a função $D\left(x, x^{\prime}\right)$ ou propagador denota a inversa do operador diferencial $\square$ no seguinte sentido:

$$
\square D(x, y)=\delta^{2}(x-y) .
$$

Neste caso, temos que

$$
\begin{aligned}
& \int d^{2} x\left[\frac{1}{2}\left(\phi+\int d^{2} y D(x, y) J(y)\right) \times\right. \\
& \square\left(\phi+\int d^{2} y D(x, y) J(y)\right) \\
& \left.-J\left(\phi+\int d^{2} y D(x, y) J(y)\right)\right] .
\end{aligned}
$$

Portanto, após algumas integrais por partes juntamente com o fato do jacobiano da transformação ser unitário, concluímos que o funcional gerador é dado por

$$
\begin{aligned}
Z[J] & =N \exp \left\{\frac{i}{2} \int d^{2} x d^{2} y J(x) D(x, y) J(y)\right\} \\
N & =\int D \phi \exp \left[-\frac{i}{2} \int d^{2} x \phi \square \phi\right] .
\end{aligned}
$$

Por fim, para encontrar o propagador proporemos o seguinte ansatz

$$
D(x, y)=\int \frac{d^{2} p}{(2 \pi)^{2}} D(p) \exp [-i p(x-y)] .
$$

A equação anterior e (33) nos conduz à identidade

$$
p^{2} D(p)=-1
$$

e, sendo assim, o propagador do campo escalar é

$$
D(x, y)=-\int \frac{d^{2} p}{(2 \pi)^{2}} \frac{1}{p^{2}} \exp [-i p(x-y)] .
$$




\subsection{O Propagador na presença de condições de contorno}

Agora precisamos implementar as condições de contorno no contexto estudado. Para isso, diremos que o campo se anule em 2 pontos distintos do espaço-tempd ${ }^{5} x^{\mu}=\left(x_{\|}, x_{\perp}\right)$

$$
\phi\left(x_{\|}, x_{\perp}=a_{k}\right)=0, \quad k=1,2 .
$$

Neste caso, essas condições de contorno podem ser implementadas no funcional gerador por meio de deltas funcionais

$$
Z_{c}=\int D \phi \prod_{k} \delta\left[\left.\phi\left(x_{\|}, x_{\perp}\right)\right|_{a_{k}}\right] \exp \left\{-i \int d^{2} x\left[\frac{1}{2} \phi \square \phi-J \phi\right]\right\}
$$

As deltas funcionais acima têm a representação funcional de Fourier dada por

$$
\delta\left[\left.\phi\left(x_{\|}, x_{\perp}\right)\right|_{a_{k}}\right]=\int D B_{k} \exp \left[-i \int d^{2} x \delta\left(x_{\perp}-a_{k}\right) B_{k}\left(x_{\|}\right) \phi\left(x_{\|}, x_{\perp}\right)\right]
$$

e, sendo assim

$$
Z_{c}=\int D \phi \int \prod_{k} D B_{k} \exp \left\{-i \int d^{2} x\left[\frac{1}{2} \phi \square \phi+\sum_{k} \delta\left(x_{\perp}-a_{k}\right) B_{k}\left(x_{\|}\right) \phi(x)-J \phi\right]\right\} .
$$

Com o intuito de desacoplar B e $\phi$, faremos a seguinte translação de campos na equação anterior

$$
\phi \rightarrow \phi-\sum_{k} \int d^{2} y \delta\left(y_{\perp}-a_{l}\right) B_{k}\left(y_{\|}\right) D(y, x)
$$

Neste caso

$$
\begin{gathered}
\int d^{2} x \frac{1}{2} \phi \square \phi \rightarrow \int d^{2} x \frac{1}{2} \phi \square \phi-\sum_{k} \int d^{2} x \delta\left(x_{\perp}-a_{k}\right) B_{k}\left(x_{\|}\right) \phi(x)+ \\
+\frac{1}{2} \sum_{k, l} \int d^{2} x d^{2} y \delta\left(x_{\perp}-a_{k}\right) B_{k}\left(x_{\|}\right) D(x, y) B_{l}\left(y_{\|}\right) \delta\left(y_{\perp}-a_{l}\right), \\
\int d^{2} x \sum_{k} \delta\left(x_{\perp}-a_{k}\right) B_{k}\left(x_{\|}\right) \phi(x) \rightarrow \sum_{k} \int d^{2} x \delta\left(x_{\perp}-a_{k}\right) B_{k}\left(x_{\|}\right) \phi(x)+ \\
-\sum_{k, l} \int d^{2} x d^{2} y \delta\left(x_{\perp}-a_{k}\right) B_{k}\left(x_{\|}\right) D(x, y) B_{l}\left(y_{\|}\right) \delta\left(y_{\perp}-a_{l}\right), \\
\int d^{2} x J \phi \rightarrow \int d^{2} x J \phi-\sum_{k} \int d^{2} x d^{2} y \delta\left(y_{\perp}-a_{k}\right) B_{k}\left(y_{\|}\right) D(y, x) J(x),
\end{gathered}
$$

e desse modo concluímos que o funcional gerador com condições de contorno é dado por

$$
\begin{aligned}
& Z_{c}=\int D \phi \prod_{k} D B_{k} \exp \left\{\frac{i}{2} \sum_{k, l} \int d^{2} x d^{2} y \delta\left(x_{\perp}-a_{k}\right) B_{k}\left(x_{\|}\right) D(x, y) B_{l}\left(y_{\perp}\right) \delta\left(y_{\perp}-a_{l}\right)+\right. \\
& \left.\left.+i \sum_{k} \int d^{2} x d^{2} y \delta\left(y_{\perp}-a_{k}\right) B_{k}\left(y_{\|}\right) D(y, x) J(x)-i \int d^{2} x\left[\frac{1}{2} \phi \square \phi-J \phi\right]\right]\right\} .
\end{aligned}
$$

\footnotetext{
$\overline{{ }^{5} \mathrm{Na} \text { notação adotada } x_{\|} \text {representa o tempo e } x_{\perp}}$ o espaço em $(1+1)$ dimensões.
} 
Podemos escrever a expressão anterior de uma maneira conveniente

$$
\begin{aligned}
& Z_{c}=\int \prod_{k} D B_{k} \exp \left\{\frac{i}{2} \sum_{k, l} \int d x_{\|} d y_{\|} B_{k}\left(x_{\|}\right) R_{k l}\left(x_{\|}, y_{\|}\right) B_{l}\left(y_{\|}\right)+\right. \\
& \left.+i \sum_{k} \int d y_{\|} B_{k}\left(y_{\|}\right) I_{k}\left(y_{\|}\right)\right\} \int D \phi \exp \left\{-i \int d^{2} x\left[\frac{1}{2} \phi \square \phi-J \phi\right]\right\}
\end{aligned}
$$

onde

$$
\begin{aligned}
& R_{k l}\left(x_{\|}, y_{\|}\right)=\int d x_{\perp} d y_{\perp} \delta\left(x_{\perp}-a_{k}\right) D(x, y) \delta\left(y_{\perp}-a_{l}\right) \\
& I_{k}\left(y_{\|}\right)=\int d y_{\perp} d^{2} x \delta\left(y_{\perp}-a_{k}\right) D(y, x) J(x) .
\end{aligned}
$$

Em notação matricial

$$
\begin{aligned}
& Z_{c}=\int \prod_{k} D B_{k} \exp \left\{\frac{i}{2} \int d x_{\|} d y_{\|} B\left(x_{\|}\right) R\left(x_{\|}, y_{\|}\right) B\left(y_{\|}\right)+i \sum_{k} \int d x_{\|} B\left(x_{\|}\right) I\left(x_{\|}\right)\right\} \times \\
& \times \int D \phi \exp \left\{-i \int d^{2} x\left[\frac{1}{2} \phi \square \phi-J \phi\right]\right\} .
\end{aligned}
$$

Agora, para desacoplar os campos das respectivas correntes na equação anterior aplicaremos as seguintes translações

$$
\begin{aligned}
& \phi \rightarrow \phi+\int d^{2} y D(x, y) J(y) \\
& B\left(x_{\|}\right) \rightarrow B\left(x_{\|}\right)-\int d y_{\|} V\left(x_{\|}, y_{\|}\right) I\left(y_{\|}\right)
\end{aligned}
$$

com a propriedade

$$
\int d y_{\|} R\left(x_{\|}, y_{\|}\right) V\left(y_{\|}, z_{\|}\right)=-\delta\left(x_{\|}-z_{\|}\right) I
$$

Somos então conduzidos ao resultado

$$
Z_{c}=N_{1} N_{2} \exp \left\{\frac{i}{2} \int d^{2} x d^{2} y J(x) D(x, y) J(y)-\frac{i}{2} \int d x_{\|} d y_{\|} I\left(x_{\|}\right) V\left(x_{\|}, y_{\|}\right) I\left(y_{\|}\right)\right\}
$$

onde definimos os determinantes funcionai:6

$$
\begin{aligned}
& N_{1}=\operatorname{det}\left[\delta^{2}(x-y) \square\right]=\int D \phi \exp \left[-\frac{i}{2} \int d^{2} x \phi \square \phi\right] \\
& N_{2}=\operatorname{det}[R]=\int \prod_{k} D B_{k} \exp \left\{\frac{i}{2} \int d x_{\|} d y_{\|} B\left(x_{\|}\right) R\left(x_{\|}, y_{\|}\right) B\left(y_{\|}\right)\right\} .
\end{aligned}
$$

Reescrevendo a equação 53 de uma maneira conveniente

$$
Z_{c}=Z[0] \exp \left\{\frac{i}{2} \int d^{2} x d^{2} y J(x)[D(x, y)-\bar{D}(x, y)] J(y)\right\}
$$

percebemos a correção ao propagador do campo escalar devido à condição de contorno imposta anteriormente. Esta é dada por

$$
\bar{D}(x, y)=\int d^{2} z d^{2} w D(x, z) \delta\left(z_{\perp}-a_{k}\right) V_{k l}\left(z_{\|}, w_{\|}\right) \delta\left(w_{\perp}-a_{l}\right) D(w, y) .
$$

\footnotetext{
$\overline{{ }^{6} \text { Para maiores detalhes sobre os determinantes funcionais } 24 .}$.
} 
Apesar de formalmente termos obtido a correção ao propagador devido à condição de contorno, temos que explicitar as equações para finalizar a análise. Neste caso, primeiramente

$$
\begin{aligned}
& R_{k l}\left(x_{\|}, y_{\|}\right)=\int d x_{\perp} d y_{\perp} \delta\left(x_{\perp}-a_{k}\right) D(x, y) \delta\left(y_{\perp}-a_{l}\right)=D\left(x_{\|}, y_{\|} ; a_{k l}\right)= \\
& =-\int \frac{d p_{\|}}{(2 \pi)} \exp \left[-i p_{\|}\left(x_{\|}-y_{\|}\right)\right] \int \frac{d p_{\perp}}{(2 \pi)} \frac{1}{p^{2}}\left[i p_{\perp} a_{k l}\right] .
\end{aligned}
$$

Resolvendo uma das integrais, concluímos que $\underbrace{7}$

$$
R_{k l}\left(x_{\|}, y_{\|}\right)=-\int \frac{d p_{\|}}{(2 \pi)} \exp \left[-i p_{\|}\left(x_{\|}-y_{\|}\right)\right] \frac{i}{2 p_{\|}} \exp \left[-i p_{\|} a_{k l}\right], \quad a_{k l}=\left|a_{k}-a_{l}\right| .
$$

Em notação matricial,

$$
\left[R\left(x_{\|}, y_{\|}\right)\right]=-\int \frac{d p_{\|}}{(2 \pi)} \exp \left[-i p_{\|}\left(x_{\|}-y_{\|}\right)\right] \frac{i}{2 p_{\|}}\left(\begin{array}{ll}
1 & e^{-i p_{\|} a} \\
e^{-i p_{\|} a} & 1
\end{array}\right) .
$$

Para encontrar a inversa de R, utilizaremos o seguinte ansatz

$$
\begin{aligned}
& {\left[V\left(y_{\|}, z_{\|}\right)\right]=\int \frac{d k_{\|}}{(2 \pi)} \exp \left[-i k_{\|}\left(y_{\|}-z_{\|}\right)\right]\left(\begin{array}{cc}
s & v \\
v & s
\end{array}\right)} \\
& \int d y_{\|}\left[R\left(x_{\|}, y_{\|}\right)\right]\left[V\left(y_{\|}, z_{\|}\right)\right]=-\delta\left(x_{\|}-z_{\|}\right) I .
\end{aligned}
$$

Portanto,

$$
\left[V\left(y_{\|}, z_{\|}\right)\right]=-\int \frac{d k_{\|}}{(2 \pi)} \exp \left[-i k_{\|}\left(y_{\|}-z_{\|}\right)\right] \frac{k_{\|}}{\operatorname{sen}\left(k_{\|} a\right)}\left(\begin{array}{cc}
e^{i k_{\|} a} & -1 \\
-1 & e^{i k_{\|} a}
\end{array}\right)
$$

Deste modo,

$$
\begin{aligned}
& \bar{D}(x, y)=\int d^{2} z d^{2} w D(x, z) \delta\left(z_{\perp}-a_{k}\right) V_{k l}\left(z_{\|}, w_{\|}\right) \delta\left(w_{\perp}-a_{l}\right) D(w, y)=\sum_{k, l} \int \frac{d k_{\|}}{(2 \pi)} \frac{d p_{\perp}}{(2 \pi)} \frac{d q_{\perp}}{(2 \pi)} \\
& V_{k l}\left(k_{\|}\right) \exp \left[-i k_{\|}\left(x_{\|}-y_{\|}\right)\right] \exp \left[i p_{\perp}\left(x_{\perp}-a_{k}\right)\right] \exp \left[i q_{\perp}\left(y_{\perp}-a_{l}\right)\right] \frac{1}{\left(k_{\|}^{2}-p_{\perp}^{2}\right)\left(k_{\|}^{2}-q_{\perp}^{2}\right)} .
\end{aligned}
$$

Escrevemos o resultado anterior em notação matricial da seguinte forma

$$
\begin{aligned}
& \bar{D}(x, y)=-\int \frac{d k_{\|}}{(2 \pi)} \exp \left[-i k_{\|}\left(x_{\|}-y_{\|}\right)\right] \frac{k_{\|}}{\operatorname{sen}\left(k_{\|} a\right)}\left(\frac{i}{2 k_{\|}} e^{i k_{\|}\left|x_{\perp}-a_{1}\right|} \frac{i}{2 k_{\|}} e^{i k_{\|}\left|y_{\perp}-a_{2}\right|}\right) \times \\
& \times\left(\begin{array}{cc}
e^{i k_{\|} a} & -1 \\
-1 & e^{i k_{\|} a}
\end{array}\right)\left(\begin{array}{c}
\frac{i}{2 k_{\|}} e^{i k_{\|}\left|x_{\perp}-a_{1}\right|} \\
\frac{i}{2 k_{\|}} e^{i k_{\|}\left|y_{\perp}-a_{2}\right|}
\end{array}\right) .
\end{aligned}
$$

Com o resultado anterior em mãos, podemos extrair resultados físicos do problema em questão.

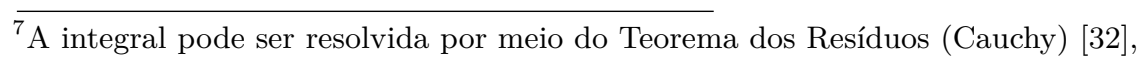

$$
\int \frac{d p_{\perp}}{(2 \pi)} \frac{1}{p^{2}}\left[i p_{\perp} a_{k l}\right]=\frac{i}{2 p_{\|}} \exp \left[-i p_{\|} a_{k l}\right], \quad a_{k l}=\left|a_{k}-a_{l}\right| .
$$




\subsection{A força de Casimir}

Neste momento estamos aptos a encontrar a energia do campo escalar real não massivo no estado de vácuo na presença das condições de contorno impostas anteriormente. Com este intuito, iniciamos com a equaçã ${ }^{8}$

$$
E=\int_{0}^{a}\langle 0|\hat{\mathcal{H}}(x)| 0\rangle d x_{\perp} .
$$

Com a ajuda da expressão da Hamiltoniana

$$
\begin{aligned}
E & =\int_{0}^{a}\left\langle 0\left|\frac{1}{2}\left[\left(\partial_{t} \hat{\phi}(x)\right)^{2}+\left(\partial_{x} \hat{\phi}(x)\right)^{2}\right]\right| 0\right\rangle d x_{\perp} \\
& =-\int_{0}^{a} \lim _{y \rightarrow x} \frac{1}{2}\left[\partial^{2}{ }_{t}+\partial^{2}{ }_{x}\right]\langle 0|\hat{\phi}(x) \hat{\phi}(y)| 0\rangle d x_{\perp}
\end{aligned}
$$

onde reescrevemos a energia a menos de derivadas totais. Utilizando a equação de movimento do campo que no caso é uma equação de onda, somos conduzidos à expressão

$$
E=-\int_{0}^{a} d x_{\perp} \lim _{y \rightarrow x} \partial^{2}{ }_{t}\langle 0|\hat{\phi}(x) \hat{\phi}(y)| 0\rangle .
$$

O valor esperado do vácuo da função de dois pontos acima pode ser calculado com o funcional gerador encontrado previamente em (55)

$$
\langle 0|\hat{\phi}(x) \hat{\phi}(y)| 0\rangle=-\frac{1}{Z_{c}(J)} \frac{\delta^{2} Z_{c}(J)}{\delta J(x) \delta J(y)}=-i[D(x, y)-\bar{D}(x, y)] .
$$

Neste caso

$$
E=i \int_{0}^{a} d x_{\perp} \lim _{y \rightarrow x} \partial^{2}{ }_{t}[D(x, y)-\bar{D}(x, y)] .
$$

Uma vez que $\mathrm{D}(\mathrm{x}, \mathrm{y})$ não depende da distância entre as placas, sua contribuição para a energia não irá produzir nenhuma força. A energia associada a $\mathrm{D}(\mathrm{x}, \mathrm{y})$ está associada à energia de vácuo livre, sem a presença das condições de contorno. Consequentemente, a energia associada a uma força é aquela que depende da distância entre as condições de contorno

$$
E=-i \int_{0}^{a} d x_{\perp} \lim _{y \rightarrow x} \partial^{2}{ }_{t} \bar{D}(x, y) .
$$

A energia de vácuo esta associada à função de Green (propagador) avaliada no mesmo ponto. Na fenomenologia de partículas de Stueckelberg-Feynman a partícula, descrita pelo propagador, é criada e destruída no mesmo ponto do espaço na representação de coordenadas. Escrevendo na representação de momentos via trasformada de Fourier, temos a criação e destruição de uma partícula com momento e energia arbitrários. Desse modo, os processos que contribuem para energia do vácuo são ditos virtuais, ou seja, na linguagem de Feynmann, temos um diagrama bolha contribuindo para energia de ponto zero.

Sendo assim a energia do campo no estado de vácuo entre as condições de contorno é dada por

$$
\begin{aligned}
& E=-i \int_{0}^{a} d x_{\perp} \int \frac{d k_{\|}}{(2 \pi)} \frac{k_{\|}}{4 \operatorname{sen}\left(k_{\|} a\right)}\left(e^{i k_{\|}\left|x_{\perp}\right|} e^{i k_{\|}\left|x_{\perp}-a\right|}\right)\left(\begin{array}{cc}
e^{i k_{\|} a} & -1 \\
-1 & e^{i k_{\|} a}
\end{array}\right)\left(\begin{array}{l}
e^{i k_{\|}\left|x_{\perp}\right|} \\
e^{i k_{\|}\left|x_{\perp}-a\right|}
\end{array}\right) \\
& E=-i \int_{0}^{a} d x_{\perp} \int \frac{d k_{\|}}{(2 \pi)} \frac{k_{\|}}{4 \operatorname{sen}\left(k_{\|} a\right)} e^{i k_{\|} a}\left[e^{2 i k_{\|} x_{\perp}}-2+e^{2 i k_{\|}\left(a-x_{\perp}\right)}\right] .
\end{aligned}
$$

Logo após algumas simplificações e integrações

$$
E=\frac{1}{4 \pi} \int i d k_{\|} \frac{k_{\|} a e^{i k_{\|} a}}{\operatorname{sen}\left(k_{\|} a\right)}
$$

\footnotetext{
${ }^{8}$ Escolhemos o sistema de referência em que $a_{1}=0$ e $a_{2}=a$
} 
Para simplificar definimos $s=a k_{\|}$

$$
E=\frac{1}{4 \pi a} \int i d s \frac{s e^{i s}}{\operatorname{sen}(s)}
$$

Agora, fazendo uma rotação de Wick $s \rightarrow i s$, observando que não temos pólos

$$
\begin{aligned}
E & =-\frac{1}{4 \pi a} \int_{-\infty}^{\infty} \frac{s e^{-s}}{\operatorname{senh}(s)} d s \\
& =-\frac{1}{2 \pi a} \int_{0}^{\infty} \frac{s e^{-s}}{\operatorname{senh}(s)} d s
\end{aligned}
$$

Calculando a integral acima somos levados ao seguinte resultadd 9

$$
E=-\frac{\pi}{24 a}
$$

Portanto, pela definição de força

$$
F=-\frac{d E}{d a}=-\frac{\pi}{24 a^{2}}
$$

que condiz com o resultado obtido pelo formalismo dos operadores.

À primeira vista, a condição de Dirichlet discutida anteriormente em (39) não parece estar associada a uma dinâmica de interação, mas sim a uma força relacionada à energia de vácuo, como podemos ver em 69). Mas, a princípio, o vácuo não possui dinâmica, sendo necessário a construção de uma dinâmica de campos que represente efetivamente a interação. Para o problema que estamos analizando é possível encontrar uma dinâmica quântica de campos (microscópica) que represente a interação efetiva de Dirichlet (macroscópica) como um caso limite, podendo então descrever o problema de interação entre os 2 objetos como sendo um problema de espalhamento 14 16]. Essa dinâmica é dada pela seguinte interação

$$
\begin{aligned}
& \mathcal{L}=\partial_{\mu} \phi \partial^{\mu} \phi-m^{2} \phi^{2}+\mathcal{L}_{i n t} \\
& \mathcal{L}_{\text {int }}=\frac{1}{2} g \sigma(x) \phi^{2}(x) \\
& \sigma(x)=\delta\left(x-\frac{a}{2}\right)+\delta\left(x+\frac{a}{2}\right) .
\end{aligned}
$$

onde foi colocado o termo de massa m para reguralizar as divergências no infravermelho e $\sigma(x)$ seria um campo externo.

Como consequência dessa interação de campos moldada em termos de um campo externo, encontramos uma força

$$
F(a, g, m)=-\frac{g^{2}}{\pi} \int_{m}^{\infty} \frac{t^{2} d t}{\sqrt{t^{2}-m^{2}}} \frac{e^{-2 a t}}{4 t^{2}+4 g t+g^{2}\left(1-e^{-2 a t}\right)}
$$

No limite de $m \rightarrow 0$ e $g \rightarrow \infty$ recuperamos o caso de Dirichlet.

\section{Leitura de Schwinger}

Apesar de utilizarmos anteriormente no formalismo funcional uma interpretação fenomenológica de partículas advinda da álgebra de operadores de criação e destruição, Schwinger constrói em contrapartida uma leitura que seja independente dessa álgebra [13,29. Para isso, ele utiliza o processo de duas fontes para descrever uma interação efetiva. A fenomenologia de Schwinger se baseia no estudo da eletrodinâmica em meios materiais 30 31 e a construção de uma dinâmica efetiva entre os objetos macroscópicos do ponto de vista de uma teoria de espalhamento via método variacional 34,35 .

A conexão da fenomenologia da interação entre meios materiais na eletrodinâmica com o nosso problema é dada pela seguinte analogia

$$
\begin{aligned}
& \vec{P}(\text { polarização }) \longrightarrow J(\text { fonte }) \\
& \vec{E}(\text { campo elétrico }) \longrightarrow \phi(\text { campo })
\end{aligned}
$$

\footnotetext{
${ }^{9} \mathrm{~A}$ integral é dada por

$$
\int_{0}^{\infty} \frac{s}{e^{s} \operatorname{senh}(s)} d s=\frac{\pi^{2}}{12}
$$
}




$$
\left.H_{\text {interação }}=-\frac{1}{2} \int d^{3} \vec{x} \vec{E} \cdot \vec{P} \text { (Hamiltoniana }\right) \longrightarrow S_{\text {interação }}=\frac{1}{2} \int d^{2} x J \phi(\text { ação }) .
$$

A princípio, considera-se uma amplitude de transição vácuo-vácuo dada em termos de uma fonte externa $\mathrm{J}$ em uma região caracterizada por uma interação, por exemplo, associada às condições de contorno definidas em 39

$$
Z[J]=\left\langle 0_{+} \mid 0_{-}\right\rangle=Z[0] e^{i S[J]}
$$

onde

$$
\begin{aligned}
& S[J]=\frac{1}{2} \int d^{2} x d^{2} y J(x) G(x, y) J(y) \\
& Z[0]=\frac{1}{\left[\operatorname{det} G^{-1}\right]^{\frac{1}{2}}}
\end{aligned}
$$

sendo $\mathrm{S}[\mathrm{J}]$ a ação efetiva e $\mathrm{G}$ a função de Green do problema em questão. A função de Green $\mathrm{G}$ apesar de ser apresentada de maneira geral (abstrata) vai depender da teoria microscópica (dinâmica, condições de contorno) escolhida.

A relação entre campos e fontes é dada por

$$
\phi(x)=\frac{1}{Z} \frac{\delta Z[J]}{i \delta J(x)}=\frac{\delta \ln (Z)}{i \delta J(x)}=\frac{\delta S}{\delta J(x)}=\int d^{2} y G(x, y) J(y) .
$$

Neste caso, podemos escrever uma expansão na amplitude de transição

$$
\begin{aligned}
& \frac{Z[J]}{Z[0]}=e^{i \int d^{2} x J(x) \phi(x)}=1+i \int d^{2} x J(x) \phi(x)+\frac{1}{2}\left[i \int d^{2} x J(x) \phi(x)\right]^{2}+\ldots \\
& \phi(x)=\frac{\delta S}{\delta J(x)} \Rightarrow S[J]=\int d^{2} x J(x) \phi(x) .
\end{aligned}
$$

Observa-se que a expansão é dada em termos da ação de interação, ou seja, todos os processos de espalhamento associados à amplitude de transição anterior foram moldados pela dinâmica de interação entre fontes dipolares e seus respectivos campos.

Agora suponha que quando perturbamos a geometria do sistema, movendo uma das condições de contorno infinitesimalmente, S é modificado, pelo menos, da seguinte forma 13. 30

$$
\delta S=\frac{1}{2} \int d^{2} x d^{2} y J(x) \delta G(x, y) J(y)
$$

ou seja, a função de Green varia com a distância quebrando a simetria de translação espacial.

Considerando que a variação em S possa ser descrita efetivamente como um processo de interação entre duas fontes dipolares induzidas e seus respectivos campos

$$
i \delta S=\frac{1}{2}\left[i \int d^{2} x J(x) \phi(x)\right]^{2},
$$

ao compararmos a equação anterior com $(83)$, somos levados ao resultado

$$
\left.i \phi(x) \phi(y)\right|_{\text {efetivo }}=\delta G(x, y) .
$$

O processo de duas fontes pode ser visto como uma interação efetiva do tipo dipólo-dipólo induzido $(J J \rightarrow \vec{P} \vec{P})$.

Sendo a energia do sistema físico escrita por uma expressão do tipo

$$
E=\int_{0}^{a} d x_{\perp} \lim _{y \rightarrow x} \partial^{2}{ }_{t} \phi(x) \phi(y)
$$


podemos afirmar que pequenas variações na distância entre as placas implicam efetivamente em um deslocamento na energia

$$
\begin{aligned}
\delta E & =\left.\int_{0}^{a} d x_{\perp} \lim _{y \rightarrow x} \partial^{2}{ }_{t} \phi(x) \phi(y)\right|_{\text {efetivo }} \\
& =-i \int_{0}^{a} d x_{\perp} \lim _{y \rightarrow x} \partial^{2}{ }_{t} \delta G(x, y),
\end{aligned}
$$

devido ao aparecimento ou desaparecimento de dipólos induzidos, idealizados pelas condições de contorno, que gerariam campos efetivos. Logo as condições de contorno (Dirichlet), representariam de maneira idelializada o surgimento de dipólos induzidos e a interação entre os mesmos, contribuindo para o deslocamento da energia. Portanto, como um exemplo, ao calcularmos a variação de 69$)$ devido a pequenas variações na distância

$$
\delta E=-i \int_{0}^{a} d x_{\perp} \lim _{y \rightarrow x} \partial^{2}{ }_{t} \delta \bar{D}(x, y)
$$

e ao compararmos com a equação (87) somos conduzidos ao resultado

$$
\delta G(x, y)=\delta \bar{D}(x, y) .
$$

Logo, o deslocamento da energia está associado à correção ao propagador devido às condições de contornq ${ }^{10}$

Alternativamente é de nosso conhecimento que

$$
\int d^{2} z G^{-1}(x, z) G(z, y)=\delta(x-y)
$$

Deste modo

$$
J(x)=\int d^{2} y G^{-1}(x, y) \phi(y)
$$

Variando 90

$$
\int d^{2} z \delta G^{-1}(x, z) G(z, y)=-\int d^{2} z G^{-1}(x, z) \delta G(z, y)
$$

concluímos

$$
\begin{aligned}
\delta G^{-1}(x, w) & =-\int d^{2} z d^{2} w G^{-1}(x, z) \delta G(z, y) G^{-1}(y, w) \\
\delta G(w, y) & =-\int d^{2} x d^{2} z G(w, x) \delta G^{-1}(x, z) G(z, y) .
\end{aligned}
$$

Consequentemente,

$$
\delta S=\frac{1}{2} \int d^{2} x d^{2} y J(w) \delta G(w, y) J(y)=-\frac{1}{2} \int d^{2} x d^{2} z \phi(x) \delta G^{-1}(x, z) \phi(z)
$$

Novamente, comparando a variação de S escrita em termos de $G^{-1}$ com o processo de duas fontes temos

$$
\left.i J(x) J(y)\right|_{\text {efetivo }}=-\delta G^{-1}(x, y) .
$$

Tendo em vista o conhecimento de Teorias Efetivas [33

$$
Z[J]=Z[0] e^{i S[J]}
$$

\footnotetext{
${ }^{10}$ Físicamente quando as placas condutoras perfeitas estão muito distantes não há dipolos induzidos. A medida que as placas vão se aproximando há o aparecimento de momento de dipólos induzidos nas placas, representado pelas fontes efetivas. Essas fontes efetivas geram campos efetivos, e a interação entre campos efetivos e fontes efetivas promovem um deslocamento da energia e a eventual interpretação de força.
} 


$$
Z[J]=\exp \{-i E[J] T\}
$$

Na equação anterior T é o tempo macroscópico associado à existência da configuração e E é a energia neste regime estacionário.

Para complementar

$$
\begin{aligned}
\left\langle 0_{+} \mid 0_{-}\right\rangle_{J} & =\left\langle 0\left|U\left(T, t_{0}\right) U\left(t_{0}, 0\right)\right| 0\right\rangle_{J} \\
& =\left\langle 0\left|e^{i \hat{H} T}\right| 0\right\rangle_{J},
\end{aligned}
$$

onde $U$ é o operador de evolução e

$$
\hat{H}|0\rangle_{J}=E[J]|0\rangle_{J}
$$

Sendo assim,

$$
\begin{aligned}
& E[J]=E[0]+E_{s}[J] \\
& E[0]=\frac{i}{T} \ln \{Z[0]\} \\
& E_{s}[J]=-\frac{1}{T} S[J] .
\end{aligned}
$$

Portanto,

$$
\begin{aligned}
\delta E_{s}[J] & =-\frac{1}{T} \delta S \\
& =-\left.\frac{1}{2} \int d^{2} x d^{2} y G(x, y) J(x) J(y)\right|_{\text {efetivo }} \\
& =-\frac{i}{2 T} \int d^{2} x d^{2} y G(x, y) \delta G^{-1}(x, y)=-\frac{i}{2 T} \operatorname{tr} \delta \ln G^{-1}
\end{aligned}
$$

onde tr é a operação de traço funciona: 11 .

Como vemos, da equação anterior é possível extrair a força de Casimir de uma dinâmica de fontes

$$
F=-\frac{\delta E_{s}[J]}{\delta a}=\frac{i}{2 T} \operatorname{tr} \frac{\delta \ln G^{-1}}{\delta a} .
$$

Usualmente encontramos a força de Casimir por meio da configuração de mínima energia

$$
\begin{aligned}
\delta E[0] & =\frac{i}{T} \delta \ln \{Z[0]\} \\
& =-\frac{i}{2 T} \delta \ln \left\{\operatorname{det}\left[G^{-1}\right]\right\} \\
& =-\frac{i}{2 T} \operatorname{tr} \delta \ln G^{-1}
\end{aligned}
$$

pois

$$
\ln \left\{\operatorname{det}\left[G^{-1}\right]\right\}=\operatorname{Tr} \ln \left\{G^{-1}\right\}
$$

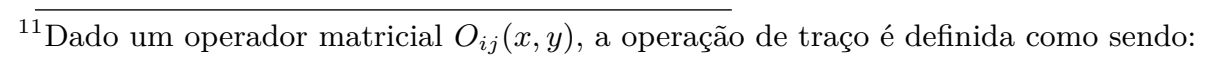

$$
\operatorname{tr} O=\sum_{i} \int d^{2} x O_{i i}(x, x) .
$$


A justificativa da passagem anterior se baseia no fato de estarmos lidando com um problema de auto-valores e auto-funções, satisfazendo as condições de contorno propostas (Dirichlet). Sendo assim, a matriz $G^{-1}$ deve possuir as seguintes propriedades

$$
\begin{aligned}
& G^{-1} \vec{\lambda}_{i}=\lambda_{i} \vec{\lambda}_{i}, \quad \operatorname{det}\left[G^{-1}-\lambda I\right]=0, \quad \vec{\lambda}_{i}=W e_{i} \Rightarrow\left(W^{-1} G^{-1} W\right) e_{i}=\lambda_{i} e_{i}, \\
& \operatorname{det}\left[W^{-1} G^{-1} W\right]=\operatorname{det}\left[G^{-1}\right]=\prod_{i} \lambda_{i}, \quad \operatorname{tr}\left[W^{-1} G W\right]=\operatorname{tr}\left[G^{-1}\right]
\end{aligned}
$$

em que $\vec{\lambda}_{i}$ representa as auto-funções, $\lambda_{i}$ os auto-valores e $e_{i}$ a base de auto-funções que diagonalizam $G^{-1}$. Então

$$
\ln \left[\operatorname{det} G^{-1}\right]=\ln \left[\prod_{i} \lambda_{i}\right]=\sum_{i} \ln \left[\lambda_{i}\right]=\operatorname{tr} \ln G^{-1} .
$$

Neste caso a configuração de mínima energia $\mathrm{J}=0$ (vácuo) juntamente com os processos virtuais estaria associada a uma dinâmica real (força). Por outro lado, na leitura de Schwinger, em que não anulamos a fonte, por trás da força de Casimir estaria uma dinâmica quântica de campos que seria descrita efetivamente como uma dinâmica de fontes macroscópicas. Como vemos as equações (101) e (102) garantem que o método para encontrar a energia mínima no formalismo canônico/funcional é equivalente ao método variacional de Schwinger.

Por fim, discutiremos sobre alguns objetos funcionais de grande importância na discussão dos fenômenos quânticos. Como vimos anteriormente $\mathrm{Z}$ é conhecido como o funcional gerador e gera toda a estrurura quântica (funções de Green) da teoria. De Z podemos definir o gerador das funções de Green conexas W

$$
Z=\exp [i W]
$$

e de W, podemos definir o gerador das funções de green irredutíveis (1PI) $\Gamma$ por meio de uma transformação de Legendre

$$
\Gamma=W-\int d^{2} x \phi J
$$

Observe que

$$
\begin{aligned}
& \int d^{2} y \frac{\delta^{2} \Gamma}{\delta \phi(x) \delta \phi(y)} \frac{\delta^{2} W}{\delta J(y) \delta J(z)}=i \delta^{2}(x-z) \\
& \frac{\delta^{2} \Gamma}{\delta \phi(x) \delta \phi(y)}=G^{-1}(x, y) .
\end{aligned}
$$

Portanto, a equação anterior nos leva a escrever a força de Casimir em termos de $\Gamma$

$$
F=\frac{i}{2 T} \operatorname{tr} \frac{\delta}{\delta a} \ln \frac{\delta^{2} \Gamma}{\delta \phi \delta \phi}
$$

condizendo com o fato de estarmos lidando com um problema de espalhamento, pois a secção de choque, que possui implicitamente o conceito de força, sempre é escrita em termos das funções de Green irredutíveis.

Partindo da equação (109) e utilizando as propriedades em (105), podemos encontrar a força de Casimir no caso da condição de contorno de Dirichlet para o campo de KGF não massivo em (1+1) dimensões da seguinte forma

$$
\begin{aligned}
F & =\frac{i}{2 T} \frac{\delta}{\delta a}\left[\sum_{i} \ln \left(\lambda_{i}\right)\right] \\
& =\left.\frac{i}{2 T} \frac{\delta}{\delta a}\left[\sum_{i}\left(\lambda_{i}\right)^{-s} \ln \left(\lambda_{i}\right)\right]\right|_{s=0} \\
& =\left.\frac{i}{2 T} \frac{\delta}{\delta a}\left[-\sum_{i} \frac{d}{d s}\left(\lambda_{i}\right)^{-s}\right]\right|_{s=0}
\end{aligned}
$$


em que vemos o surgimento da função zeta generalizada 23

$$
\left.\left[\sum_{i} \frac{d}{d s}\left(\lambda_{i}\right)^{-s}\right]\right|_{s=0} \doteq \zeta\left(G^{-1}, s=0\right) .
$$

Logo com a ajuda dos conceitos e cálculos abordados em 22 podemos concluir que,

$$
F=-\frac{i}{2 T} \frac{\delta \zeta\left(G^{-1}, s=0\right)}{\delta a}=-\frac{\pi}{24 a^{2}} .
$$

\section{Conclusões e perspectivas futuras}

Como vimos, apresentamos duas interpretações ao efeito Casimir, a primeira advinda da fenomenologia de partículas associada à álgebra dos operadores de criação e destruição e, a segunda advinda da fenomenologia associada à força entre dielétricos e a necessidade de uma dinâmica.

$\mathrm{Na}$ interpretação de criação e destruição de partículas, a força de Casimir, uma quantidade física real, está sendo interpretada como advinda de processos virtuais não físicos. Sendo assim, o vácuo possui essa dinâmica de processos virtuais escondida no princípio da incerteza. Porém com a operação de ordenamento normal, sabemos que o vácuo não participa da dinâmica, pois ele não tem dinâmica. Dessa forma, ao apresentarmos a linguagem funcional para descrever o fenômeno de Casimir percebemos que ao visualizarmos o processos que contribuem para energia via fenomenologia de Stueckelberg-Feynmann, temos diagramas bolha, partículas criadas e destruídas no mesmo ponto do espaço-tempo (na representação de coordenadas) e com energia e momento arbitrários (na representação de momentos).

Em contrapartida, na leitura de Schwinger, a força de Casimir está associada à interação efetiva entre duas fontes advinda da fenomenologia de campos eletromagnéticos em meios materiais e vemos a relação entre a força e as funções de Green irredutíveis, percebendo que estamos lidando com um espalhamento (Matriz-S) de dois objetos macroscópicos. Sendo assim, temos uma dinâmica quântica de campos entre os 2 objetos. Por fim, sem a necessidade de visualizar os processos quânticos (Schwinger), também foi possível moldar uma dinâmica quântica de campos que descrevesse efetivamente a força de Casimir, encontrando uma lagrangiana de interação com campos externos. Portanto, na abordagem conceitual de Schwinger ao se estudar a força de Casimir escolhemos uma dinâmica microscópica (interação via campos externos, fontes) que descreve efetivamente uma dinâmica macroscópica (Dirichilet), sem a necessidade de interpretar o fenômeno em termos da energia de vácuo (modos normais) com sua dinâmica de processos virtuais não físicos, mas sim em termos de uma interação quântica do tipo van der Waals (dipólo-dipólo).

É válido ressaltar que apesar de descrevermos o efeito Casimir distante da realidade física, impondo condições de contorno no campo escalar de KleinGordon-Fock não massivo em $(1+1)$ dimensões, temos toda a metodologia e os aspectos principais para abordar o problema na Eletrodinâmica Quântica em $(1+3)$ dimensões. Também podemos estender o estudo, implementando os ingredientes estatísticos necessários para descrever o fenômeno, estudando o problema à temperatura finita $(T \neq 0)$ com os campos em equilíbrio termodinâmico [36]. A abordagem de uma dinâmica estatística microscópica efetiva enriqueceria a descrição do efeito Casimir, discutido ao longo do texto à temperatura $(T=0)$.

\section{Agradecimentos}

F. A. Barone agradece o $\mathrm{CNPq}$ pelo suporte financeiro parcial, A. A. Nogueira agradece a CAPES pelo suporte financeiro total e B. M. Pimental agradece a CAPES e CNPq pelo suporte financeiro parcial. Os autores agradecem ao parecerista anônimo pela revisão cuidadosa do artigo e observações que tornaram o texto mais claro e didático.

\section{Referências}

[1] H.B.G. Casimir and D. Polder, Phys. Rev 73, 4 (1948).

[2] H.B.G. Casimir, Proc. K. Ned. Akad. Wet. 51, 793 (1948). 
[3] Marcus Venicius Cougo-Pinto, Carlos Farina e Alexandre Tort, Rev. Bras. Ens. Fis. 22, 1 (2000).

[4] S.L. Boersma, Am. J. Phys 64, 539 (1996).

[5] S.K. Lamoreaux, Phys. Rev. Lett 78, 5 (1997); and the erratum, 81, 5475 (1998).

[6] M.J. Sparnaay, Physica 24, 6 (1958).

[7] G.L. Klimchitskaya, U. Mohideen and V.M. Mostepanenko, Rev. Mod. Phys. 81, 1827 (2009).

[8] E.M. Lifshitz, Soviet Physics JETF 2, 1 (1956).

[9] S.Y. Buhmann, Dispersion Forces I. Macroscopic Quantum Electrodynamics and Ground-State Casimir, Casimir-Polder and van der Waals Forces (Springer Tracts in Modern Physics, London, 2012), 1st ed.

[10] S.Y. Buhmann, Dispersion Forces II. Many-Body Effects, Excited Atoms, Finite Temperature and Quantum Friction (Springer Tracts in Modern Physics, London, 2012), 1st ed.

[11] L.A. Ferreira, Sobre a Energia de Ponto Zero do Campo Eletromagnético na Presença de Meios Materiais. Master's Thesis, IFT, São Paulo (1980).

[12] M. Bordag, K. Kirsten and D.V. Vassilevichz,J. Phys. A: Math. Gen. 31, 2381 (1998).

[13] J. Schwinger, Lett. Math. Phys. 1, 43 (1975).

[14] N. Graham, R.L. Jaffe, V. Khemani, M. Quandt, M. Scandurrab and H. Weigel, Nucl. Physics. B 645, 49 (2002).

[15] N. Grahan, R.L. Jaffe, V. Khemani, M. Quandt, M. Scandurra and H. Weigel, Phys. Lett. B 572, 196 (2003).

[16] R.L. Jaffe,Phys.Rev D 72, 021301 (2005).

[17] M. Bordag, U. Mohideen and V.M. Mostepanenko, Phys. Rep. 353, 1 (2001).

[18] K.A. Milton, The Casimir Effect: Physical Manifestations of Zero-Point Energy (World Scientific, New Jersey, 2001), 1st ed.

[19] M. Bordag, G.L. Klimchitskaya, U. Mohideen and V.M. Mostepanenko, Advances in the Casimir Effect (Oxford University Press, New York, 2009), 1st ed.
[20] K.A. Milton and M. Bordag, Quantum Field Theory under the Influence of External Conditions (World Scientific, New Jersey, 2010), 1st ed.

[21] F.A.B. Rangel, Correções Radiativas em Teoria Quântica de Campos sob Condições de Contorno. P.h.D Thesis, UFRJ/IF, Rio de Janeiro (2003).

[22] J.J.P. Sobrinho e A.C. Torty, Rev. Bras. Ens. Fis. 23, 4 (2001).

[23] E. Elizalde, S.D. Odintsov, A. Romeo, A.A. Bytsenko and S. Zerbini, Zeta Regularization Techniques with Applications (World Scientific, Singapore, 1994), 1st ed.

[24] W. Greiner and J. Reinhardt, Field Quantization (Springer, Berlin, 1996), 1st ed.

[25] J. Schwinger, Phys. Rev. 82, 5 (1951).

[26] G. Barton and K. Schanhorst, J. Phys. A: Math. Gen. 26, 2037 (1993).

[27] M. Blazhyevska, Journal of Physical Studies 16, 3001 (2012).

[28] M. Bordag, D. Robaschik and E. Wieczorek, Ann. Phys 165, 192 (1985).

[29] J. Schwinger, Particles, Sources and Fields (Perseus Books, Massachusetts, 1973), v. I, II and III, 1st ed.

[30] P.W. Milonni, The Quantum Vacuum: An Introduction to Quantum Electrodynamics (Academic Press, New York, 1994), 1st ed.

[31] K.A. Milton, arXiv: 9811054 (1998).

[32] G.B. Arfken and H.J. Weber, Mathematical Methods for Physicists (Academic Press, New York, 1995), 4 th ed.

[33] S. Weinberg, The Quantum Theory of Fields (Cambridge University Press, 1996), v. II, 1st en.

[34] J. Schwinger, Symbolism of Atomic Measurements (Springer, New York 2001), 1st ed.

[35] C.A.M. de Melo , B.M. Pimentel e J.A. Ramirez, Rev. Bras. Ens. Fis. 35, 4 (2013).

[36] T. Matsubara, Progr. Theor. Phys. 14, 351 (1955); E.S. Fradkin, Selected Papers on Theoretical Physics, edited by I.V. Tyutin (Lebedev Institute, Moscow, 2007). 